\title{
Orbital motion of the massive multiple stars in the Orion Trapezium
}

\author{
D. Schertl ${ }^{1}$, Y. Y. Balega ${ }^{2}$, Th. Preibisch ${ }^{1}$, and G. Weigelt ${ }^{1}$ \\ 1 Max-Planck-Institut für Radioastronomie, Auf dem Hügel 69, 53121 Bonn, Germany \\ 2 Special Astrophysical Observatory, Nizhnij Arkhyz, Zelenchuk region, Karachai-Cherkesia, 357147, Russia
}

Received 12 August 2002 / Accepted 6 February 2003

\begin{abstract}
We present bispectrum speckle interferometry of the multiple Orion Trapezium stars $\theta^{1}$ Ori A, $\theta^{1}$ Ori $\mathrm{B}$, and $\theta^{1}$ Ori C obtained with the SAO 6 m telescope in Russia over a period of 5.5 years (epochs 1995-2001). Our diffraction-limited images have a resolution $\lambda / D$ of 42 mas ( $J$-band), 57 mas $(H$-band) and 76 mas ( $K$-band). We clearly detect motion of the companions relative to their primary stars in the systems $\theta^{1}$ Ori A1-2 (mean separation $\rho \sim 220$ mas, change in position angle $\Delta \mathrm{PA}=6^{\circ}$ ), $\theta^{1}$ Ori B2-3 $\left(\rho \sim 205\right.$ mas, $\left.\Delta \mathrm{PA}=8^{\circ}\right)$, and $\theta^{1}$ Ori $1-2\left(\rho \sim 37\right.$ mas, $\left.\Delta \mathrm{PA}=18^{\circ}\right)$. In our $K$-band image of $\theta^{1}$ Ori $\mathrm{B}$ we resolve a fourth visual component, confirming its discovery by Simon et al. (1999). We determine the $J, H$, and $K$ magnitudes of the system components and estimate the stellar masses of the companions in the HR-diagram. The companions $\theta^{l}$ Ori $\mathrm{C} 2$ and $\theta^{1}$ Ori B2 show clear evidence of near-infrared excess in the color-color diagram. The companions $\theta^{1}$ Ori A2 and $\theta^{1}$ Ori B 3 show much stronger extinction than their primary stars, providing evidence of the presence of circumstellar material around the companions.
\end{abstract}

Key words. techniques: interferometric - stars: individual: $\theta^{1}$ Ori C, $\theta^{1}$ Ori A, $\theta^{1}$ Ori B - stars: binaries: general stars: pre-main sequence

\section{Introduction}

It is well known that most of the stars in our galaxy are in multiple systems. The binary frequency is about $50 \%$ for low-mass stars (see Duquennoy \& Mayor 1991; Fischer \& Marcy 1992) and seems to be significantly higher $(>70 \%)$ for the more massive O- and B-type stars (see Mason et al. 1998; Abt et al. 1990; Preibisch et al. 1999; García \& Mermilliod 2001). The high multiplicity of massive stars, and in particular the large number of very close systems (see e.g. Mermilliod \& García 2001), begs important questions about the formation mechanism of massive stars, which is still not well understood (cf. Stahler et al. 2000; Yorke \& Sonnhalter 2002). A particularly interesting model in this context is the scenario described by Bonnell et al. (1998), in which massive stars form through accretioninduced collisions and subsequent merging of protostars in the dense central regions of forming stellar clusters. This model predicts a high frequency of close multiple systems (i.e. failed mergers) among the massive stars, which is supported by recent observational results.

The Orion Nebula is one of the most prominent and nearest $(D \sim 450 \mathrm{pc})$ star forming regions and contains a particularly dense cluster of very young $\left(<1 \times 10^{6} \mathrm{yr}\right)$ stars (see e.g. Herbig \& Terndrup 1986; McCaughrean \& Stauffer 1994;

Send offprint requests to: Th. Preibisch, e-mail: preib@mpifr-bonn.mpg.de
Hillenbrand 1997). The Trapezium system $\left(\theta^{1}\right.$ Ori ABCD), four massive and luminous O-type and early B-type stars, is located in the center of the cluster. The strong stellar wind and the ionizing radiation of the most massive cluster member $\theta^{1}$ Ori $\mathrm{C}$ have strong effects on the surrounding cloud material (Bally et al. 1998; see also Richling \& Yorke 1998).

In Weigelt et al. (1999) and Preibisch et al. (1999) we presented the results of a bispectrum speckle interferometric survey for multiple systems among $13 \mathrm{O}$ - and B-type Orion Nebula cluster members. We found 8 new visual companions, increasing the number of known visual and spectroscopic companions of the 13 target stars to 14. A particularly interesting result of our speckle observations was the discovery of a close $(\sim 16 \mathrm{AU})$ visual companion to $\theta^{1}$ Ori C. We also confirmed earlier detections of visual companions to $\theta^{1}$ Ori A (Petr et al. 1998) and $\theta^{1}$ Ori B (Simon et al. 1999).

In this paper we present the results of repeated bispectrum speckle interferometry observations of the Trapezium stars $\theta^{1}$ Ori $\mathrm{A}, \theta^{1}$ Ori $\mathrm{B}$, and $\theta^{1}$ Ori $\mathrm{C}$ obtained over a period of 5.5 years. We discuss the relative motion in the systems and derive new mass estimates for the components from multi-color near-infrared photometry. An important aim of our study is a better characterization of the physical properties of the massive multiple systems, which may ultimately allow us to draw conclusions on their formation mechanisms. 
Table 1. Observational parameters. $\lambda_{c}$ and $\Delta \lambda$ give the central wavelength and bandwidth of the filter. In the detector column, $H$ denotes HAWAII array detector, and P the PICNIC detector. $N_{\mathrm{T}}$ and $N_{\mathrm{R}}$ are the numbers of speckle interferograms of the object and reference-star, respectively. $T$ is the exposure time per frame and $S$ the seeing $(F W H M)$. In the last column, the names of the reference stars are given.

\begin{tabular}{|c|c|c|c|c|c|c|c|c|c|}
\hline $\begin{array}{l}\theta^{1} \\
\text { Ori }\end{array}$ & Epoch & $\begin{array}{l}\lambda_{\mathrm{c}} \\
{[\mathrm{nm}]}\end{array}$ & $\begin{array}{l}\Delta \lambda \\
{[\mathrm{nm}]}\end{array}$ & $\begin{array}{c}\text { Detector / } \\
\text { Pixel size } \\
\text { [mas] }\end{array}$ & $\overline{N_{\mathrm{T}}}$ & $N_{\mathrm{R}}$ & $\begin{array}{c}T \\
{[\mathrm{~ms}]}\end{array}$ & $\begin{array}{c}S \\
{\left[{ }^{\prime \prime}\right]}\end{array}$ & $\begin{array}{l}\text { Reference } \\
\text { Star }\end{array}$ \\
\hline A & 1995.775 & 2191 & 411 & $\mathrm{P} / 31.8$ & 97 & 97 & 100 & 1.8 & $\theta^{1}$ Ori $\mathrm{E}$ \\
\hline A & 1996.247 & 2191 & 411 & P / 30.9 & 403 & 403 & 250 & 2.2 & $\theta^{1}$ Ori E \\
\hline A & 1996.746 & 2191 & 411 & P / 30.6 & 547 & 540 & 200 & 2.4 & $\theta^{1}$ Ori D \\
\hline A & 1997.788 & 2165 & 328 & $\mathrm{P} / 30.5$ & 753 & 388 & 200 & 2.0 & $\theta^{1}$ Ori $\mathrm{E}$ \\
\hline A & 1998.838 & 2165 & 328 & $\mathrm{H} / 27.0$ & 385 & 385 & 120 & 3.4 & $\theta^{1}$ Ori E \\
\hline A & 1999.715 & 2115 & 214 & $\mathrm{H} / 27.0$ & 173 & 173 & 160 & 1.2 & $\theta^{1}$ Ori E \\
\hline A & 1999.737 & 1239 & 138 & $\mathrm{H} / 13.4$ & 340 & 340 & 100 & 1.8 & $\theta^{1}$ Ori E \\
\hline A & 2000.765 & 2115 & 214 & $\mathrm{H} / 27.0$ & 140 & 140 & 160 & 3.4 & $\theta^{1}$ Ori E \\
\hline A & 2000.781 & 1648 & 317 & $\mathrm{H} / 20.2$ & 86 & 86 & 80 & 1.8 & $\theta^{1}$ Ori E \\
\hline A & 2000.781 & 1239 & 138 & H / 13.4 & 85 & 85 & 60 & 2.0 & $\theta^{1}$ Ori E \\
\hline A & 2001.186 & 2115 & 214 & $\mathrm{H} / 27.0$ & 435 & 435 & 240 & 1.4 & $\theta^{1}$ Ori E \\
\hline B & 1996.249 & 2191 & 411 & $\mathrm{P} / 30.9$ & 379 & 379 & 250 & 2.4 & $\theta^{1}$ Ori $\mathrm{E}$ \\
\hline B & 1996.746 & 2191 & 411 & P / 30.6 & 526 & 540 & 200 & 2.2 & $\theta^{1}$ Ori D \\
\hline B & 1997.784 & 1613 & 304 & $\mathrm{P} / 19.7$ & 425 & 300 & 150 & 2.2 & $\theta^{1}$ Ori D \\
\hline B & 1997.792 & 2191 & 411 & $\mathrm{P} / 30.5$ & 1057 & 730 & 200 & 2.0 & $\theta^{1}$ Ori D \\
\hline B & 1998.838 & 2165 & 328 & $\mathrm{H} / 27.0$ & 385 & 385 & 120 & 3.4 & $\theta^{l}$ Ori E \\
\hline B & 1999.715 & 2115 & 214 & $\mathrm{H} / 27.0$ & 173 & 173 & 160 & 1.2 & $\theta^{1}$ Ori E \\
\hline B & 1999.737 & 1239 & 138 & $\mathrm{H} / 13.4$ & 351 & 344 & 100 & 1.8 & $\theta^{1}$ Ori $\mathrm{E}$ \\
\hline B & 2000.765 & 2115 & 214 & $\mathrm{H} / 27.0$ & 140 & 140 & 160 & 3.4 & $\theta^{1}$ Ori E \\
\hline B & 2001.186 & 2115 & 214 & H / 27.0 & 435 & 435 & 240 & 1.4 & $\theta^{1}$ Ori E \\
\hline $\mathrm{C}$ & 1997.784 & 1613 & 304 & $\mathrm{P} / 19.7$ & 519 & 641 & 150 & 2.2 & $\theta^{1}$ Ori $\mathrm{D}$ \\
\hline $\mathrm{C}$ & 1998.838 & 2115 & 214 & $\mathrm{H} / 27.0$ & 438 & 265 & 120 & 3.6 & $\theta^{1}$ Ori D \\
\hline $\mathrm{C}$ & 1999.737 & 1239 & 138 & H / 13.4 & 516 & 244 & 100 & 1.4 & $\theta^{1}$ Ori D \\
\hline $\mathrm{C}$ & 2001.184 & 1239 & 138 & $\mathrm{H} / 13.4$ & 684 & 1523 & 80 & 3.6 & $\theta^{1}$ Ori D \\
\hline
\end{tabular}

\section{Observations and data reduction}

The speckle interferograms were recorded with the SAO $6 \mathrm{~m}$ telescope in Russia between 1995 and 2001. The detector of our speckle camera was either a Rockwell HAWAII array (19982001 ; only one $512 \times 512$ quadrant was used) or a $256 \times 256$ PICNIC array detector (1995-1997). Further observational parameters are listed in Table 1.

Diffraction-limited images were reconstructed using the bispectrum speckle interferometry method (Weigelt 1977; Weigelt \& Wirnitzer 1983; Lohmann et al. 1983; Hofmann \& Weigelt 1986). The object power spectrum was determined with the speckle interferometry method (Labeyrie 1970). Speckle interferograms of unresolved single stars were recorded just before and just after the object and served as reference stars for the determination of the speckle transfer function.

The plate scale and orientation of the detectors were derived from all available observations of wide calibration binaries $\left(\theta^{1}\right.$ Ori ABE, ADS 3734, 11558, 19159, 14575, 14504, 10184). The plate scale and orientation errors were determined to be $0.6 \%$ and $0.4^{\circ}$, respectively.

\section{Results}

In Fig. 1 we show our speckle images of $\theta^{1}$ Ori $\mathrm{A}, \theta^{1}$ Ori $\mathrm{B}$, and $\theta^{1}$ Ori $C$ : all have close companions. The orientation, separation, and flux ratio of the close companion of $\theta^{1}$ Ori $\mathrm{C}$ were determined by fitting cosine functions to the speckle interferometry power spectrum. For $\theta^{1}$ Ori A and $\theta^{1}$ Ori B, these parameters were determined using aperture photometry. The parameters are listed in Table 2.

In our new image of $\theta^{1}$ Ori B we clearly see the fourth visual component, $\theta^{1}$ Ori B4, originally detected by Simon et al. (1999). In our image we find a separation of $\rho=609 \pm 8$ mas and a brightness ratio of $\Delta K=5.05 \pm 0.8 \mathrm{mag}$; these values are in reasonable agreement with the parameters $\rho=573$ mas and $\Delta K=4.26$ mag derived by Simon et al. (1999) from their adaptive optics image. This component was not visible in our earlier data simply due to inadequate signal-to-noise.

\subsection{Physical companions or chance projections?}

Without additional information, we cannot be sure that the stars we observe near to the OB stars are indeed physically related to the primaries: there might also be chance projections of 
Table 2. Parameters of the speckle companions derived from our images.

\begin{tabular}{|c|c|c|c|c|c|c|}
\hline$\theta^{1}$ Ori & Epoch & $\begin{array}{l}\text { Separation } \\
{[\mathrm{mas}]}\end{array}$ & $\begin{array}{l}\text { PA } \\
{[\mathrm{deg}]}\end{array}$ & Flux ratio & $\begin{array}{l}\lambda_{\mathrm{c}} \\
{[\mathrm{nm}]}\end{array}$ & $\begin{array}{l}\Delta \lambda \\
{[\mathrm{nm}]}\end{array}$ \\
\hline \multirow[t]{11}{*}{ A1-2 } & 1995.775 & $227 \pm 5$ & $350.6 \pm 2$ & $0.30 \pm 0.02$ & 2191 & 411 \\
\hline & 1996.247 & $227 \pm 4$ & $352.8 \pm 2$ & $0.29 \pm 0.02$ & 2191 & 411 \\
\hline & 1996.746 & $223 \pm 4$ & $252.7 \pm 2$ & $0.30 \pm 0.02$ & 2191 & 411 \\
\hline & 1997.788 & $224 \pm 4$ & $353.0 \pm 2$ & $0.24 \pm 0.02$ & 2165 & 328 \\
\hline & 1998.838 & $221 \pm 5$ & $353.8 \pm 2$ & $0.25 \pm 0.02$ & 2165 & 328 \\
\hline & 1999.715 & $219 \pm 3$ & $355.4 \pm 2$ & $0.26 \pm 0.02$ & 2115 & 214 \\
\hline & 1999.737 & $215 \pm 3$ & $354.8 \pm 2$ & $0.12 \pm 0.02$ & 1239 & 138 \\
\hline & 2000.765 & $215 \pm 4$ & $356.2 \pm 2$ & $0.23 \pm 0.02$ & 2115 & 214 \\
\hline & 2000.781 & $216 \pm 4$ & $356.1 \pm 2$ & $0.23 \pm 0.02$ & 1648 & 317 \\
\hline & 2000.781 & $211 \pm 4$ & $356.0 \pm 2$ & $0.15 \pm 0.02$ & 1239 & 138 \\
\hline & 2001.186 & $215 \pm 3$ & $356.6 \pm 2$ & $0.25 \pm 0.02$ & 2115 & 214 \\
\hline \multirow[t]{9}{*}{ B1-2 } & 1996.249 & $955 \pm 10$ & $255 \pm 1$ & $0.31 \pm 0.02$ & 2191 & 411 \\
\hline & 1996.746 & $944 \pm 10$ & $253 \pm 1$ & $0.34 \pm 0.02$ & 2191 & 411 \\
\hline & 1997.784 & $942 \pm 10$ & $255 \pm 1$ & $0.12 \pm 0.02$ & 1613 & 304 \\
\hline & 1997.792 & $941 \pm 10$ & $255 \pm 1$ & $0.29 \pm 0.02$ & 2191 & 411 \\
\hline & 1998.838 & $942 \pm 10$ & $254 \pm 1$ & $0.30 \pm 0.02$ & 2165 & 328 \\
\hline & 1999.715 & $940 \pm 10$ & $255 \pm 1$ & $0.27 \pm 0.02$ & 2115 & 214 \\
\hline & 1999.737 & $941 \pm 10$ & $254 \pm 1$ & $0.043 \pm 0.02$ & 1239 & 138 \\
\hline & 2000.773 & $938 \pm 10$ & $255 \pm 1$ & $0.30 \pm 0.02$ & 2115 & 214 \\
\hline & 2001.186 & $942 \pm 10$ & $255 \pm 1$ & $0.20 \pm 0.02$ & 2115 & 214 \\
\hline \multirow[t]{9}{*}{ B2-3 } & 1996.249 & $122 \pm 5$ & $202 \pm 3$ & $0.34 \pm 0.04$ & 2191 & 411 \\
\hline & 1996.746 & $118 \pm 5$ & $202 \pm 3$ & $0.27 \pm 0.04$ & 2191 & 411 \\
\hline & 1997.784 & $114 \pm 5$ & $204 \pm 3$ & $0.40 \pm 0.04$ & 1613 & 304 \\
\hline & 1997.792 & $118 \pm 5$ & $204 \pm 3$ & $0.31 \pm 0.04$ & 2191 & 411 \\
\hline & 1998.838 & $117 \pm 5$ & $206 \pm 3$ & $0.32 \pm 0.05$ & 2165 & 328 \\
\hline & 1999.715 & $116 \pm 4$ & $206 \pm 2$ & $0.30 \pm 0.04$ & 2115 & 214 \\
\hline & 1999.737 & $116 \pm 3$ & $207 \pm 2$ & $0.57 \pm 0.03$ & 1239 & 138 \\
\hline & 2000.773 & $113 \pm 5$ & $209 \pm 3$ & $0.30 \pm 0.04$ & 2115 & 214 \\
\hline & 2001.186 & $117 \pm 4$ & $210 \pm 3$ & $0.25 \pm 0.03$ & 2115 & 214 \\
\hline B1-4 & 2001.186 & $609 \pm 8$ & $298 \pm 2$ & $0.0095 \pm 0.01$ & 2115 & 214 \\
\hline \multirow[t]{4}{*}{$\mathrm{C} 1-2$} & 1997.784 & $33 \pm 3$ & $226 \pm 5$ & $0.26 \pm 0.02$ & 1613 & 304 \\
\hline & 1998.838 & $37 \pm 4$ & $222 \pm 5$ & $0.32 \pm 0.03$ & 2115 & 214 \\
\hline & 1999.737 & $43 \pm 2$ & $214 \pm 3$ & $0.31 \pm 0.02$ & 1239 & 138 \\
\hline & 2001.184 & $38 \pm 2$ & $208 \pm 3$ & $0.29 \pm 0.02$ & 1239 & 138 \\
\hline
\end{tabular}

unrelated sources. The probability of such a chance projection can be determined from the luminosity function of the stars in this field and the magnitude of the supposed companions, all of which ${ }^{1}$ are brighter than $K=9$. According to the $K$ band luminosity function of the Trapezium region given by Simon et al. (1999), the probability of finding a chance projected star with $K \leq 9$ within $1^{\prime \prime}$ of a given position is $0.4 \%$. This estimate is based on the luminosity function for the whole cluster, whereas most of the brightest sources are concentrated close to the cluster center. However, even if we count all known objects in the innermost 15" radius area (McCaughrean \& Stauffer 1994), including the various (supposed) companions to the massive stars, the probability of finding a chance projected star with $K \leq 9$ within $1^{\prime \prime}$ of a given position is only $4.4 \%$. Since nearly all of our observed companions are at much smaller separations from their primary stars than $1^{\prime \prime}$, we are confident that the companions we observe are actually physically related to the corresponding primary. Nevertheless,

\footnotetext{
1 With the exception of the faint component B4, which, however, is not considered in the analysis below.
}

the possibility of a chance projection cannot be completely ruled out, and will be considered in the analysis below.

In the unlikely case of a chance projection of two unrelated stars, one would expect to see relative motion due to the proper motion dispersion of the cluster. Jones \& Walker (1988) measured the relative proper motions for over 1000 stars in the Orion Nebula and found the 2D velocity dispersion to be $1.5 \mathrm{mas} / \mathrm{yr}$. The proper motion distribution is quite broad, but most stars show proper motions within the range of $\pm \sim 3.5 \mathrm{mas} / \mathrm{yr}$. Over the 5.5 year period covered by our measurements, displacements would typically be $\sim 8$ mas and up to $\sim 20$ mas.

\subsection{Relative motion in the multiple systems}

In Fig. 2 we show our relative astrometry measurements for the four systems, $\theta^{1}$ Ori A1-2, $\theta^{1}$ Ori B1-2, $\theta^{1}$ Ori B2-3, and $\theta^{1}$ Ori $C 1-2$. Our data clearly show relative motion in three of the four observed systems. 

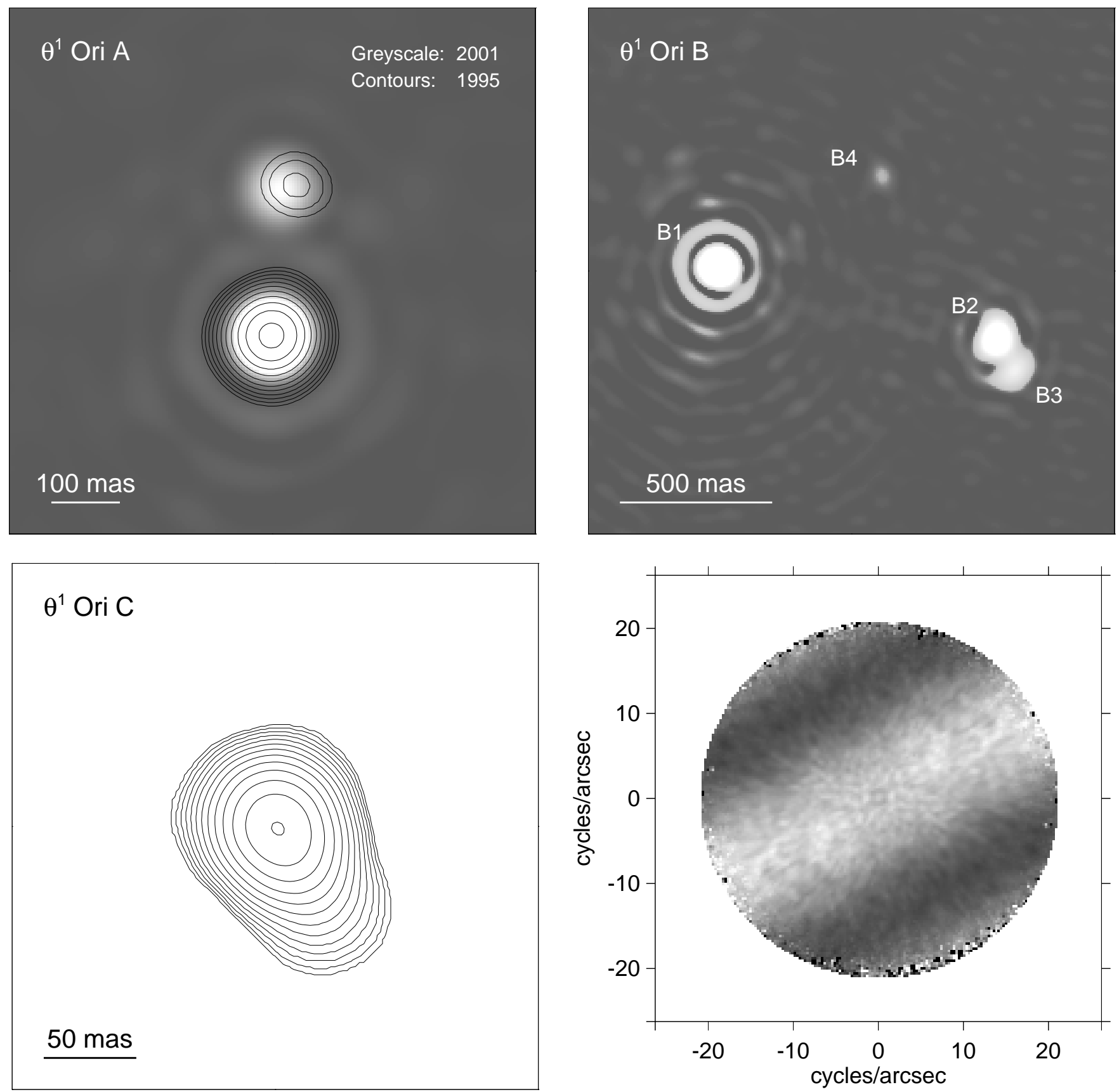

Fig. 1. Upper left: bispectrum speckle $K$-band images of $\theta^{1}$ Ori A. The greyscale image was reconstructed from the 2001 data, the contour image from the 1995 data. The orbital motion of the companion can be easily seen. Upper right: bispectrum speckle $K$-band image of $\theta$ Ori B (2001 data). The faint fourth component is seen near the center of the upper half of the image. Lower left: contour representation of our $J$-band bispectrum speckle image of $\theta^{1}$ Ori C (2001 data). Lower right: reconstructed power spectrum of $\theta^{1}$ Ori C ( $J$ band, 2001 data).

For $\theta^{1}$ Ori A1-2 (Fig. 2a), the position angle increased by $6^{\circ}$ during the $5.5 \mathrm{yr}$ period of our observations, while the separation decreased from $\sim 227$ mas (102 AU) to $\sim 215$ mas (97 AU). If, as we believe, the companion is actually physically bound to the primary star, this suggests either a circular orbit inclined with respect to the line-of-sight, or an elliptical orbit. However, the relative motion is also consistent with a straight line (as expected for proper motion in a chance projected system), and the measured angular velocity is consistent with the proper motion dispersion of the cluster. Thus, there is no clear proof of a bound system as yet.
The system $\theta^{1}$ Ori B2-3 also shows clear motion: the position angle increased by $8^{\circ}$ within $5 \mathrm{yr}$, while the separation increased from 202 mas to 210 mas, again suggesting a tilted circular or an elliptical orbit. Once more, however, the observed angular velocity is consistent with the proper motion dispersion of the cluster, and thus a chance projection cannot be completely ruled out.

Clear evidence of relative motion is also seen in the visual system $\theta^{1}$ Ori $\mathrm{C} 1-2$, where the position angle increased by $18^{\circ}$ within $3.4 \mathrm{yr}$. Here, the possibility of a chance projection seems extremely unlikely, due to the very small separation and the 

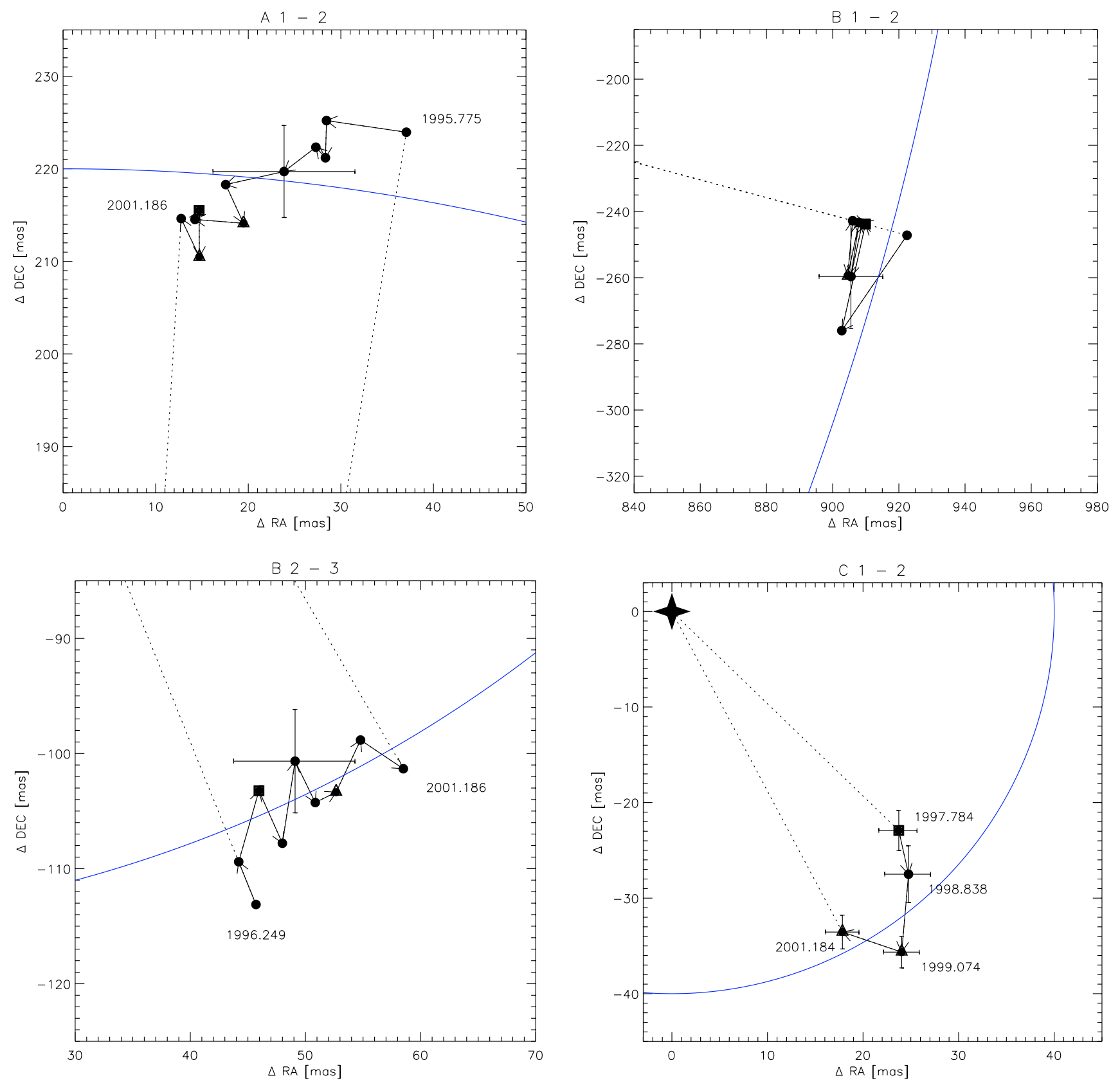

Fig. 2. Relative motion in the systems $\theta^{1}$ Ori A1-2, $\theta^{1}$ Ori B1-2, $\theta^{1}$ Ori B2-3, and $\theta^{1}$ Ori $C 1-2$. The brighter component is always placed at the origin $(0,0)$ of the plot. The solid symbols represent the observed positions of the companion over time from Table 2; $J$-band data are shown by triangles, $H$-band data by squares, and $K$-band data by circles. For clarity we show error bars in each plot for one data point only. In the plot for $\theta^{1}$ Ori $\mathrm{C} 1-2$ we show error bars for all four orbital points, and we also mark the primary position by a big asterisk. In each plot the first and last positional measurements in the data series are connected to the origin by a thin dotted line. The solid line shows a part of a circle around the primary position.

brightness of the companion ${ }^{2}$, and because the observed motion is apparently inconsistent with a straight line.

No significant relative motion is detected for the system $\theta^{1}$ Ori B1-2, although this is not very surprising, given the relatively wide apparent separation of about 425 AU.

\footnotetext{
2 Based on the magnitude distribution in the innermost $15^{\prime \prime}$ radius area of the Trapezium cluster (McCaughrean \& Stauffer 1994), the statistical probability that a chance projection with an unrelated star might mimic a companion as bright and close as observed is only $8 \times$ $10^{-6}$.
}

As we are dealing with measurements at different wavelengths, we have to take into consideration the following potential problem: if a star is surrounded by an asymmetrical halo of nongray dust, then its photocenter might be wavelengthdependent. Under very unfavorable circumstances this effect might mimic orbital motion. Furthermore, in the case of the Trapezium cluster circumstellar material can be photoionized by the central OB stars, creating asymmetric "proplyds" (see Bally et al. 1998), which, due to their emission-line nature, 


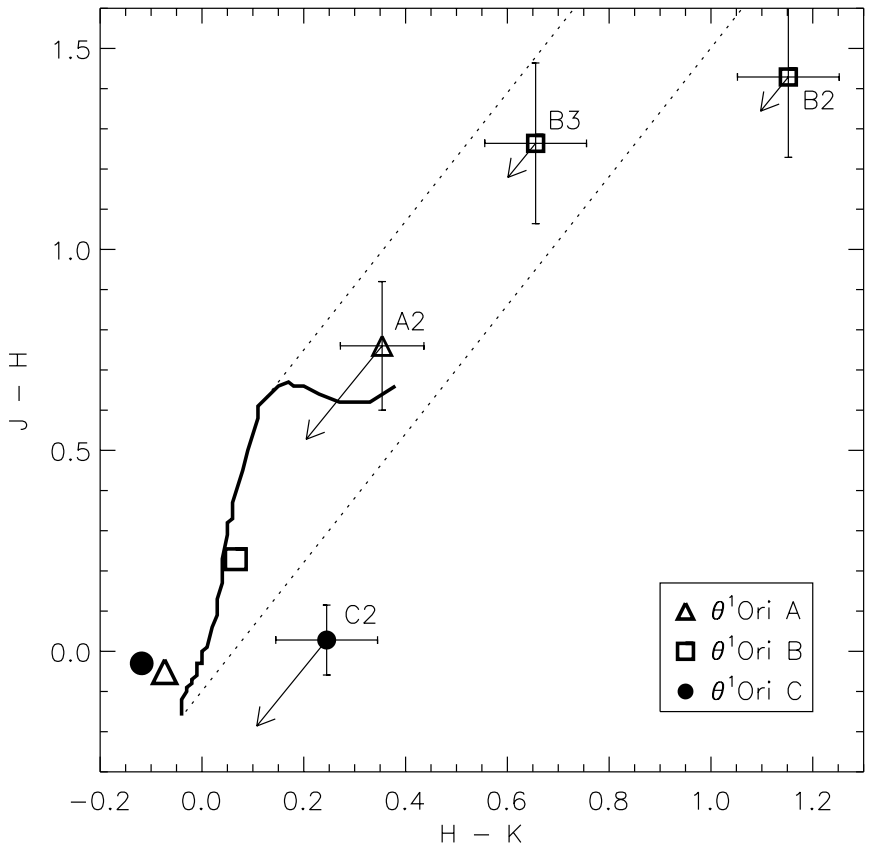

Fig. 3. Near-infrared color-color diagram for the components of the Trapezium stars. The large symbols show the de-reddened colors of the primary stars $\theta^{1}$ Ori A, $\theta^{1}$ Ori B, and $\theta^{1}$ Ori C. The small symbols with error-bars show the derived colors of the companions; the arrows represent the inverse reddening vectors (using the extinction law tabulated by Mathis 1990 for $R_{V}=5$ ) for the extinction of the corresponding primary star. The solid line shows the photospheric colors for main sequence stars, the dotted lines indicate the reddening band for photospheric colors.

differ significantly in brightness when observed through different broad-band filters.

In order to see whether our data might be affected by this effect, we plotted the orbit points in Fig. 2 with different symbols (triangles, squares, and circles) for the three different wavelengths ( $J, H$, and $K$ band). We find no significant systematic differences in the positions at different wavelengths and, therefore, believe that our data are unaffected by this potential problem.

\subsection{Near-infrared magnitudes of the components and stellar mass estimates}

From published photometric data for the unresolved systems and the flux ratios measured in our speckle images, we determined the near-infrared magnitudes of the individual components. For the systems $\theta^{1}$ Ori $\mathrm{A}$ and $\theta^{1}$ Ori $\mathrm{C}$ we use the photometric data compiled by Hillenbrand et al. (1998). For $\theta^{1}$ Ori B we use data in the 2MASS catalog, which we assume here to represent the combined fluxes of the components ${ }^{3} \mathrm{~B} 1, \mathrm{~B} 2$, and B3. In Fig. 3 we show the $J-H$ versus $H-K$ color-color diagram for the components of $\theta^{1}$ Ori A, $\theta^{1}$ Ori B, and $\theta^{1}$ Ori C. This diagram is useful for looking for infrared excesses and for estimating the extinction of the stars.

\footnotetext{
${ }^{3}$ We neglect the contribution of the faint fourth visual component B4.
}

Table 3. Near-infrared magnitudes of the system components and photometric estimates of the extinction and the stellar masses. The extinction and mass values for the primary components are taken from Hillenbrand et al. (1998).

\begin{tabular}{llllrr}
\hline \hline$\theta^{1}$ Ori & $J$ & $\begin{array}{l}H-K \\
{[\mathrm{mag}]}\end{array}$ & $\begin{array}{c}J-H \\
{[\mathrm{mag}]}\end{array}$ & $\begin{array}{r}A_{V} \\
{[\mathrm{mag}]}\end{array}$ & $\begin{array}{r}M_{\star} \\
{[\mathrm{mag}]}\end{array}$ \\
\hline A1 & 6.14 & 0.08 & 0.18 & 1.89 & 16 \\
A2 & 8.45 & 0.35 & 0.76 & 3.8 & $\approx 4$ \\
\hline B1 & 6.82 & 0.12 & 0.31 & 0.69 & 7 \\
B2 & 10.23 & 1.15 & 1.43 & $\gtrsim 9$ & $\approx 4$ \\
B3 & 10.84 & 0.66 & 1.26 & 7.8 & $\approx 3$ \\
\hline C1 & 4.63 & 0.02 & 0.18 & 1.74 & 45 \\
C2 & 6.22 & 0.25 & 0.03 & & $\gtrsim 6$ \\
\hline
\end{tabular}

$\theta^{1}$ Ori A 2 is located well within the reddening band, i.e. it displays colors consistent with pure photospheric emission without infrared excess. The extinction can be obtained by moving the object backwards along the direction of the reddening vector until it crosses the photospheric color line. The colors of $\theta^{1}$ Ori A2 allow, in principle, two different solutions of the de-reddening problem: the star might be either an M-type star extincted by only $A_{V} \sim 1.5 \mathrm{mag}$, or an $\sim$ F-type star extincted by $A_{V} \sim 3.8 \mathrm{mag}$. This ambiguity can be resolved by taking into account the observed brightness of the object and its corresponding location in a color-magnitude diagram or an HR-diagram (see Fig. 4). Such an analysis shows that the first alternative (M-type star with low extinction) is not plausible, since the observed brightness is much larger than predicted by PMS evolution models, even for extremely young objects; in the HR-diagram, the star would be located far above the birthline. The second alternative, i.e. a strongly extincted $\sim$ F-type star, yields a reasonable location in the HR-diagram, suggesting the object to be a very young intermediate-mass PMS star.

$\theta^{1}$ Ori B2 is located to the right of the reddening band, i.e. in the infrared excess region. This clearly indicates the presence of relatively hot circumstellar material. The excess makes it very difficult to derive an accurate value of the object's extinction; our best guess is an $A_{V}$ of around 9 mag. The presence of infrared excess is not surprising, as $\theta^{1}$ Ori B2 is associated with a proplyd and a jet. The bright, one-sided jet can be seen in the HST images of Bally et al. (1998). Bally et al. (2000) discuss this microjet, with the name HH 508 or $\mathrm{j} 160-307$, in detail. It originates from $\theta^{1}$ Ori B2, extends about $0.5^{\prime \prime}$ along position angle $317^{\circ}$, and represents the brightest Herbig-Haro object $^{4}$ in the entire Orion nebula, but was not previously recognized because of its proximity to $\theta^{1}$ Ori B1. $\theta^{1}$ Ori $\mathrm{B} 2$ is also associated with a faint and very compact proplyd tail, which points directly away from $\theta^{1}$ Ori B1 (position angle $255^{\circ}$ ). The direction of the proplyd tail and the exceptional surface brightness of the jet suggest that it is physically close to $\theta^{1}$ Ori B1.

${ }^{4}$ The microjet has an exceptional surface brightness because it is externally ionised (cf. Reipurth et al. 1998). 
This supports our assumption that the objects $\theta^{1}$ Ori B1-2-3 are actually physically related and not just a chance projection.

$\theta^{1}$ Ori B3 is located within the reddening band. Using similar arguments as above for $\theta^{1}$ Ori A2, the de-reddening procedure suggests the object to be an $\sim$ F-type star extincted by $A_{V} \sim 8 \mathrm{mag}$.

$\theta^{1}$ OriC2 is located in the excess region, suggesting the presence of circumstellar material. Given the excess and the relatively blue colors, it is not possible to determine the extinction of this star.

Summarizing these results, we find evidence of significant amounts of circumstellar matter around all four companion stars: for $\theta^{1}$ Ori $\mathrm{C} 2$ and $\theta^{1}$ Ori B2 the circumstellar material is traced directly by the strong near-infrared excesses (and the proplyd from $\theta^{1}$ Ori B2); in the cases of $\theta^{1}$ Ori A2 and $\theta^{1}$ Ori B3, the extinction of the putative companion is much larger than that of the corresponding primary star, i.e. it must be local circumstellar extinction.

The masses of the companions of the Trapezium stars were estimated in Weigelt et al. (1999), using only the $K$ and $H$-band flux ratios available at that time. The additional information now available through our $J$-band observations allows a better estimation of the extinctions, and thus enables us to make better founded mass estimates. Here, we use the same procedure as in our earlier study, but we base our analysis on the $J$-band magnitudes and the $J-H$ colors, because these wavelengths are less affected than the $K$ band by infrared excesses from circumstellar material. The de-reddened $J$-band magnitudes of the companions can be transformed into a stellar luminosity as a function of the (unknown) stellar temperature using the compilation of intrinsic $V-J$ colors and bolometric corrections of Kenyon \& Hartmann (1995). The $J$-band magnitude of each star thus defines a line in the HR diagram. On the other hand, the de-reddened $J-H$ color can be transformed into the stellar temperature. Given the uncertainties in the nearinfrared magnitudes of the companions, this procedure yields a more or less extended band of locations in the HR-diagram, as shown in Fig. 4. These bands can now be compared to PMS evolutionary models, in order to estimate stellar masses. Given the uncertainties in the colors derived from the flux-ratios, it seems useful to take two further constraints into account: we expect the position of the YSOs in the HR-diagram to be below the birthline (see Palla \& Stahler 1999) and above the $1 \mathrm{Myr}$ isochrone ${ }^{5}$.

For $\theta^{1}$ Ori $\mathrm{A} 2$ the band in the HR diagram is rather well constrained and suggests a mass of $\sim 4 M_{\odot}$. In the case of $\theta^{1}$ Ori B2, which shows a strong near-infrared excess, we cannot constrain the intrinsic colors. Our best guess for this mass is $M \sim 4 M_{\odot}$. The color for $\theta^{1}$ Ori B 3 is rather well constrained, leading to a mass estimate of $\sim 3 M_{\odot}$.

The near-infrared excess of $\theta^{1}$ Ori $C 2$ prevents us from deriving accurate colors of the star; however, we believe it is safe to assume that the intrinsic $J-H$ color is not redder than the observed color. This implies the spectral type to be no later than about A0. This (relatively weak) criterion corresponds to

5 This is a firm upper limit on the age of the Trapezium stars; see Prosser et al. (1994).
Table 4. This table lists the estimated masses of the multiple systems, the mean observed separation $\langle\rho\rangle$, and the expected Keplerian orbital period $P_{\circ}$, assuming a circular orbit. We also list the expected changes in position angle during the period of our observations for the case of a pole-on view onto the orbital plane, $\Delta \mathrm{PA}_{\text {exp }}$, and the observed position angle changes, $\triangle \mathrm{PA}_{\mathrm{obs}}$.

\begin{tabular}{lrrrrr}
\hline \hline $\begin{array}{l}\text { system } \\
\theta^{1} \text { Ori }\end{array}$ & $\begin{array}{r}M_{\mathrm{tot}} \\
{\left[M_{\odot}\right]}\end{array}$ & $\begin{array}{r}\langle\rho\rangle \\
{[\mathrm{AU}]}\end{array}$ & $\begin{array}{r}P_{\circ} \\
{[\mathrm{yr}]}\end{array}$ & $\begin{array}{r}\Delta \mathrm{PA}_{\exp } \\
{[\mathrm{deg}]}\end{array}$ & $\begin{array}{r}\Delta \mathrm{PA}_{\mathrm{obs}} \\
{[\mathrm{deg}]}\end{array}$ \\
\hline A1-2 & 20 & 98.7 & 214 & 9.1 & 5 \\
B1-23 & $\sim 14$ & 424.3 & $\sim 2300$ & $\sim 0.8$ & $<2$ \\
B2-3 & $\sim 7$ & 52.3 & $\sim 140$ & $\sim 13$ & 8 \\
C1-2 & $\approx 51$ & 17.0 & $<9.8$ & $>125$ & 18 \\
\hline
\end{tabular}

a very extended band of possible locations in the HR diagram. We can, therefore, only derive a lower limit for the mass of the object, $M \gtrsim 6 M_{\odot}$.

We are aware that our mass estimates might be subject to significant uncertainties, especially given the intrinsic tentativeness of the theoretical PMS models.

\section{Discussion}

\subsection{Consistency of the photometric mass estimates and the orbital motion}

We can now compare the masses estimated from the photometric data with the observed relative motion in the systems and test for consistency with orbital motion. For $\theta^{1}$ Ori A1-2 the observed change in PA is about half of that expected for a circular orbit seen perpendicular to the orbital plane. This difference by a factor of two can easily be explained by projection effects or an eccentric orbit. Therefore, the observed motion is consistent with the assumption of orbital motion in a system with stellar masses as estimated from the photometry. Our non-detection of significant orbital motion in the system $\theta^{1}$ Ori B1-23 is well in agreement with the expected long period of about $2300 \mathrm{yr}$. For the system $\theta^{1}$ Ori B2-3 our period estimate agrees reasonably well with the observed motion. Only in the case of $\theta^{1}$ Ori C1-2 is the observed motion much less than expected for a pole-on view onto a circular orbit. However, the difference could easily be explained by projection effects, i.e. by a strongly inclined orbit, or by an eccentric orbit.

\subsection{Spectroscopic results for the $\theta^{1}$ Ori $C$ system}

Two recent studies found evidence for $\theta^{1}$ Ori $\mathrm{C}$ being a spectroscopic binary. Donati et al. (2002) presented spectropolarimetric observations of $\theta^{1}$ Ori $C$. They summarized the observational evidence for radial velocity variations of $\theta^{1}$ Ori $C$ and confirmed a trend of increasing radial velocity over the last decade. They concluded that these results argue strongly in favor of $\theta^{1}$ Ori $\mathrm{C}$ being a spectroscopic binary, with a period of at least $8 \mathrm{yr}$ in the case of a highly eccentric orbit and more than $16 \mathrm{yr}$ if the orbit is circular. The visual companion $\theta^{1}$ Ori $\mathrm{C} 2$ detected in our speckle images is a very good candidate for this spectroscopic 


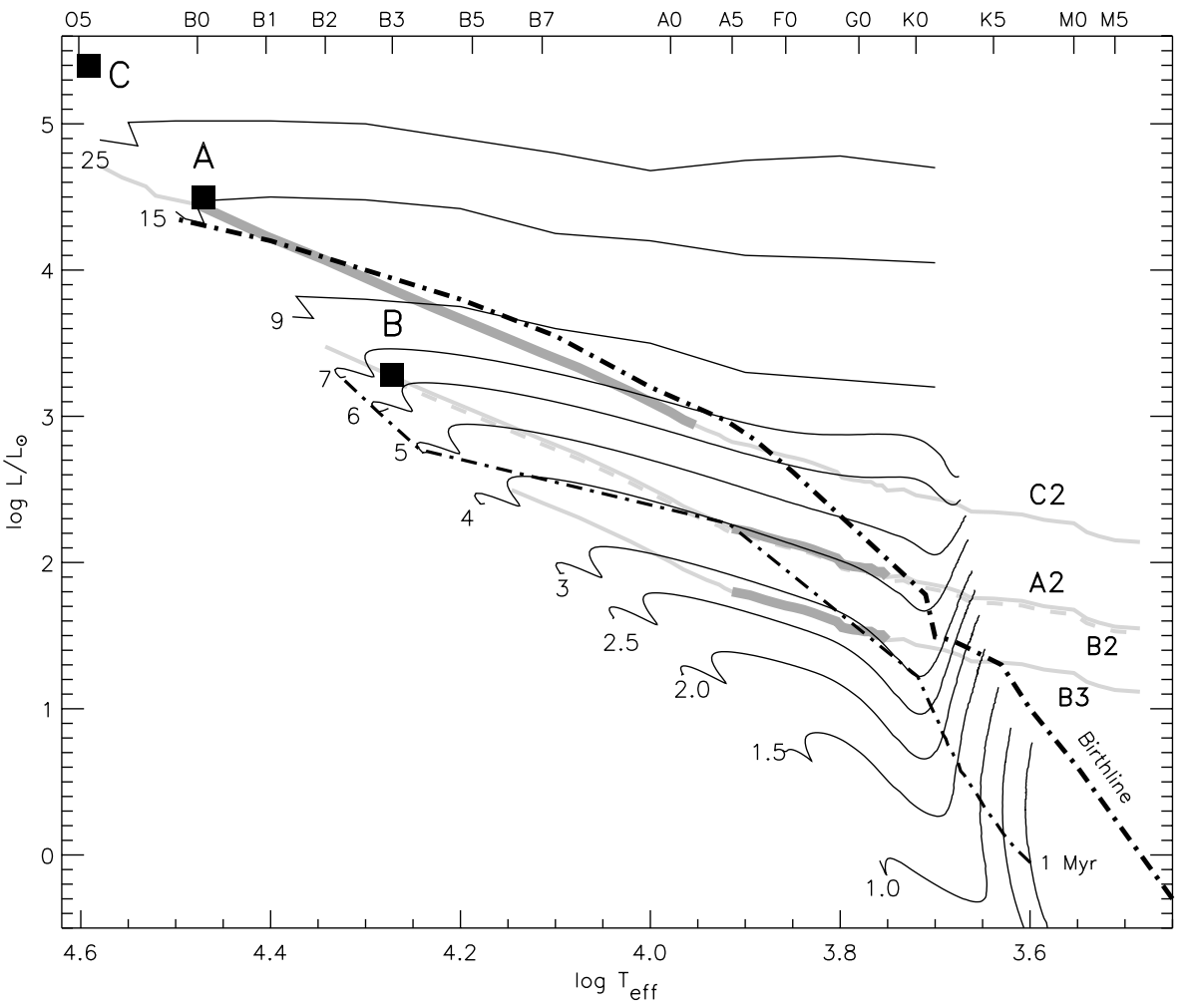

Fig. 4. HR-diagram with PMS evolutionary tracks (solid lines labeled by the corresponding masses in $M_{\odot}$ ) from Siess et al. (1997) for $M \leq 7 M_{\odot}$ and from Bernasconi $\&$ Maeder (1996) for $M \geq 9 M_{\odot}$. The upper dashed-dotted line is the stellar birthline according to Palla \& Stahler (1999), the lower dashed-dotted line shows the $1 \mathrm{Myr}$ isochrone. The positions of the primary stars are shown by the squares. The grey lines show the range of allowed locations of the speckle companions according to their $J$-band magnitudes. The thick and dark parts of these lines take into account the information derived from the $J-H$ color (see text for details). companion. Donati et al. (2002) note that if their interpretation of the radial velocity variations is correct, the radial velocities and the separation between the components should soon undergo very rapid variations.

Vitrichenko (2002) analyzed the radial velocity of $\theta^{1}$ Ori C, based on literature data and IUE spectra. From the temporal variations of the radial velocity he concluded that $\theta^{1}$ Ori $\mathrm{C}$ is a close binary with a period of 66 days. This period suggests a semimajor axis of $a \sim 1 \mathrm{AU}$, and therefore, the spectroscopic companion cannot be the speckle companion. However, Vitrichenko (2002) also claimed evidence for long-term variations of the mean radial velocity of the $\theta^{1}$ Ori $\mathrm{C}$ spectroscopic system, which he interpreted as an indication of the presence of a third component. For this third component he derived a period of $120 \mathrm{yr}$, suggesting a semimajor axis of $a \sim 80 \mathrm{AU}$. It might well be possible that the speckle companion $\theta^{1}$ Ori $\mathrm{C} 2$ is the third spectroscopic component.

Finally, although the findings of Vitrichenko (2002) differ clearly from those of Donati et al. (2002), our present data are unable to discriminate between them.

\section{Conclusions}

Our ongoing monitoring of the systems will soon enable us to (1) test whether or not we actually see orbital motion and not random motion in chance projected systems, and (2) determine the presumed orbits more accurately. The case of $\theta^{1}$ Ori $C$ is especially interesting, because a better determination of the orbit will allow us to decide which of the two models proposed on the basis of spectroscopic data (the binary model of Donati et al. 2002 or the triple model of Vitrichenko 2002) is correct. Better knowledge of the multiplicity of $\theta^{1}$ Ori $\mathrm{C}$ is also very important with respect to several peculiar properties of $\theta^{1}$ Ori C (e.g. its periodic variability, hard $\mathrm{X}$-ray emission, and magnetic field), many of which are not yet well understood.

Acknowledgements. We thank the referee Mark McCaughrean for his constructive critical report that helped to improve this paper.

\section{References}

Abt, H. A., Gomez, A. E., \& Levy, S. G. 1990, ApJS, 74, 551

Bally, J., Sutherland, R. S., Devine, D., \& Johnstone, D. 1998, AJ, 116, 293

Bally, J., O’Dell, C. R., \& McCaughrean, M. J. 2000, AJ, 119, 2919

Bernasconi, P. A., \& Maeder, A. 1996, A\&A, 307, 829

Bonnell, I. A., Bate, M. R., \& Zinnecker, H. 1998, MNRAS, 298, 93

Donati, J.-F., Babel, J., Harries, T. J., et al. 2002, MNRAS, 333, 55

Duquennoy, A., \& Mayor, M. 1991, A\&A, 248, 485

Fischer, D. A., \& Marcy, G. W. 1992, ApJ, 396, 178

García, B., \& Mermilliod, J. C. 2001, A\&A, 368, 122

Herbig, G. H., \& Terndrup, D. M. 1986, ApJ, 307, 609

Hillenbrand, L. A. 1997, AJ, 113, 1733

Hillenbrand, L. A., Strom, S. E., Calvet, N., et al. 1998, AJ, 116, 1816

Hofmann, K.-H., \& Weigelt, G. 1986, A\&A, 167, L15

Jones, B. F., \& Walker, M. F. 1988, AJ, 95, 1755

Kenyon, S. J., \& Hartmann, L. 1995, ApJS, 101, 117

Labeyrie, A. 1970, A\&A, 6, 85

Lohmann, A. W., Weigelt, G., \& Wirnitzer, B. 1983, Appl. Opt., 22, 4028

Mason, B. D., Gies, D. R., Hartkopf, W. I., et al. 1998, AJ, 115, 821

Mathis, J. S. 1990, ARA\&A, 28, 37 
Mermilliod, J.-C., \& García, B. 2001, in The Formation of Binary Stars, Proc. of IAU Symp., 200, 191

McCaughrean, M. J., \& Stauffer, J. R. 1994, AJ, 108, 1382

Palla, F., \& Stahler, S. W. 1999, ApJ, 525, 772

Petr, M. G., Coudé Du Foresto, V., Beckwith, S. V. W., Richichi, A., \& McCaughrean, M. J. 1998, ApJ, 500, 825

Preibisch, Th., Balega, Y., Hofmann, K.-H., Weigelt, G., \& Zinnecker, H. 1999, New Astron., 4, 531

Prosser, C. F., Stauffer, J. R., Hartmann, L., et al. 1994, ApJ, 421, 517

Reipurth, B., Bally, J., Fesen, R. A., \& Devine, D. 1998, Nature, 396, 343
Richling, S., \& Yorke, H. W. 1998, A\&A, 340, 408

Siess, L., Forestini, M., \& Dougados, C. 1997, A\&A, 324, 556

Simon, M., Close, L. M., \& Beck, T. L. 1999, AJ, 117, 1375

Stahler, S. W., Palla, F., \& Ho, P. T. P. 2000, in Protostars and Planets IV, ed. V. Mannings, A. P. Boss, \& S. S. Russel (Tucson: University of Arizona Press), 327

Vitrichenko, E. A. 2002, Astron. Lett., 28, 324

Weigelt, G. 1977, Opt. Commun., 21, 55

Weigelt, G., Balega, Y., Preibisch, Th., et al. 1999, A\&A, 347, L15

Weigelt, G., \& Wirnitzer, B. 1983, Opt. Lett., 8, 389

Yorke, H. W., \& Sonnhalter, C. 2002, ApJ, 569, 846 